CORRECTION

Series B

\title{
Correction to: Idealness of $k$-wise intersecting families
}

\author{
Ahmad Abdi $^{1}$ D $\cdot$ Gérard Cornuéjols $^{2} \cdot$ Tony Huynh $^{3} \cdot$ Dabeen Lee $^{4}$
}

Published online: 1 February 2022

(c) The Author(s) 2022

\section{Correction to: Mathematical Programming https://doi.org/10.1007/s10107-020-01587-x}

Let $\mathcal{C}$ be a clutter over ground set $V \cdot \mathcal{C}$ is ideal if the polyhedron $Q(\mathcal{C}):=$ $\left\{x \in \mathbb{R}_{+}^{V}: \sum_{v \in C} x_{v} \geq 1 \forall C \in \mathcal{C}\right\}$ is integral. Given an integer $k \geq 2, \mathcal{C}$ is $k$-wise intersecting if every subset of at most $k$ members have a common element, yet no element belongs to all members. In the paper, we studied the following conjecture.

Conjecture 3 There exists an integer $k \geq 4$ such that every $k$-wise intersecting clutter is non-ideal.

Let $n \geq 1$ be an integer, and let $S \subseteq\{0,1\}^{n}$. Let $\operatorname{conv}(S)$ denote the convex hull of $S$. An inequality of the form $\sum_{i \in I} x_{i}+\sum_{j \in J}\left(1-x_{j}\right) \geq 1$, for some disjoint $I, J \subseteq[n]$, is called a generalized set covering inequality. The set $S$ is cube-ideal if every facet of $\operatorname{conv}(S)$ is defined by $x_{i} \geq 0, x_{i} \leq 1$, or a generalized set covering inequality.

\section{Published in Mathematical Programming, 2020 [3].}

The original article can be found online at https://doi.org/10.1007/s10107-020-01587-x.

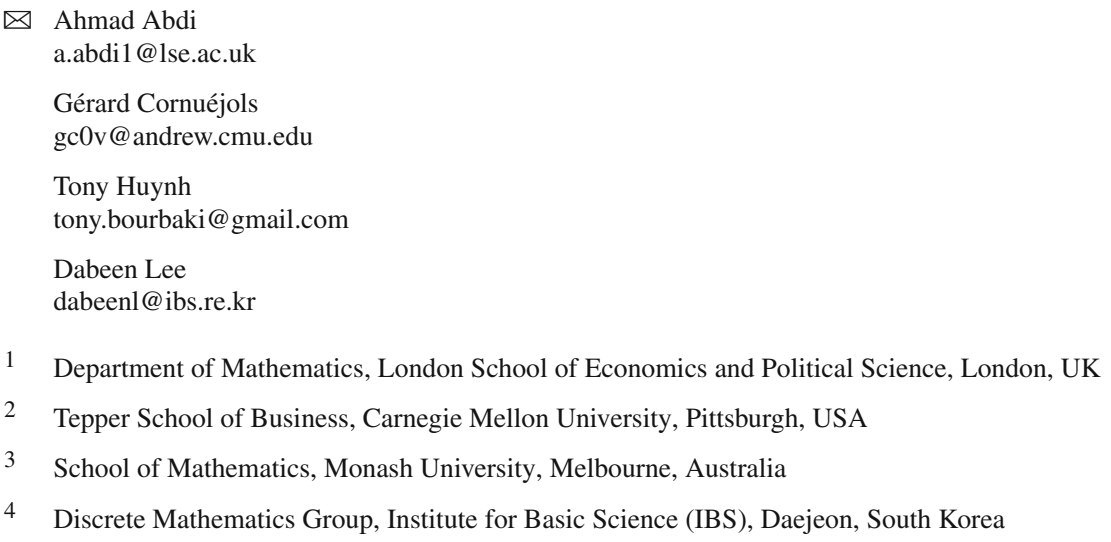


Conjecture 16 There exists an integer $k \geq 4$ such that for every cube-ideal set $S$, either all the points in $S$ agree on a coordinate, or there is a subset of $S$ of at most $k$ points that do not agree on a coordinate.

At the beginning of $\S 4$ of the paper, we showed that Conjecture 3 for $k$ implies Conjecture 16 for the same $k$. Then, in $\$ 4.2$, we proved that the two conjectures are equivalent. While the statement is true, our proof is flawed; we address the source of the flaw shortly. For now, let us provide a shorter, correct proof of this fact.

Theorem 23 Conjecture 16 for $k$ is equivalent to Conjecture 3 for the same $k$.

Revised proof We already showed $(\Leftarrow)$. It remains to prove $(\Rightarrow)$. Suppose Conjecture 3 is false for some $k \geq 4$. That is, there is an ideal $k$-wise intersecting clutter $\mathcal{C}$ over ground set $\{1,2, \ldots, n\}$. Let

$$
S:=\left\{\chi_{C}: C \subseteq[n] \text { contains a member of } \mathcal{C}\right\}
$$

Observe that $S$ is an up-monotone subset of $\{0,1\}^{n}$. By Theorem 4.3 of [2], since $\mathcal{C}$ is an ideal clutter, $S$ is a cube-ideal set. Moreover, since $\mathcal{C}$ is $k$-wise intersecting, the points in $S$ do not agree on a coordinate but every subset of $S$ of $\leq k$ points do. Therefore, $S$ refutes Conjecture 16 for $k$, as required.

The culprit. The previous proof of Theorem 23 relied on Theorem 22, but there is an oversight in the proof of that theorem. More specifically, the last two sentences of the proof of Claim 3, starting with "Thus, to finish the proof," and ending with "We leave this as an easy exercise for the reader." are incorrect. As a result, we retract the second claim of Theorem 22, as follows.

Theorem 22 Let $\mathcal{C}$ be an ideal tangled clutter. Then $\operatorname{core}(\mathcal{C})$ is a duplication of a cuboid.

In particular, we do not prove that every ideal tangled clutter has an ideal core (though this has been proved using more advanced machinery recently [1]). The only result in the paper that relies on Theorem 22 is Theorem 23, which remains correct.

Fixing Theorem 22. The proof of Theorem 22 can be expanded to show something different. Moving forward, we use terminology and results from our paper without recalling them.

Theorem 22a Let $\mathcal{C}$ be an ideal tangled clutter over ground set $V$, let $G=(V, E)$ be the bipartite graph over vertex set $V$ whose edges correspond to the minimum covers of $\mathcal{C}$, and let $\left\{U_{1}, V_{1}\right\}, \ldots,\left\{U_{r}, V_{r}\right\}$ be the bipartitions of the connected components of $G$. Then the following statements hold:

1. core $(\mathcal{C})$ is a tangled clutter.

2. For each $i \in[r]$, pick $u_{i} \in U_{i}$ and $v_{i} \in V_{i}$. Let $\mathcal{C}^{\prime}$ be the clutter over ground set $\left\{u_{1}, v_{1}, \ldots, u_{r}, v_{r}\right\}$ obtained from core $(\mathcal{C})$ after contracting $V-$ $\left\{u_{1}, v_{1}, \ldots, u_{r}, v_{r}\right\}$. Then $\operatorname{conv}\left(\left\{\chi_{C}: C \in \mathcal{C}^{\prime}\right\}\right)$ can be described by $z \geq \mathbf{0}$, $z_{u_{i}}+z_{v_{i}}=1, i \in[r]$, and

$$
\sum_{B \cap U_{i} \neq \emptyset}\left|B \cap U_{i}\right| z_{u_{i}}+\sum_{B \cap V_{j} \neq \emptyset}\left|B \cap V_{j}\right| z_{v_{j}} \geq 1
$$


for every minimal cover $B$ of $\mathcal{C}$ such that for each $i \in[r], B \cap\left(U_{i} \cup V_{i}\right) \subseteq U_{i}$ or $V_{i}$. 3. $\mathcal{C}^{\prime}$ is the cuboid of some subset $S \subseteq\{0,1\}^{r}$, where conv $(S)$ is defined by $\mathbf{0} \leq x \leq \mathbf{1}$, and

$$
\sum_{B \cap U_{i} \neq \emptyset}\left|B \cap U_{i}\right| x_{i}+\sum_{B \cap V_{j} \neq \emptyset}\left|B \cap V_{j}\right|\left(1-x_{j}\right) \geq 1
$$

for every minimal cover $B$ of $\mathcal{C}$ such that for each $i \in[r], B \cap\left(U_{i} \cup V_{i}\right) \subseteq U_{i}$ or $V_{i}$.

Proof By Proposition 20 (3), for each $i \in[r]$, the elements in $U_{i}$ are duplicates in core $(\mathcal{C})$, the elements in $V_{i}$ are duplicates in $\operatorname{core}(\mathcal{C})$, and $|\{u, v\} \cap C|=1$ for all $u \in U_{i}, v \in V_{i}$ and $C \in \operatorname{core}(\mathcal{C})$. That is, each $C \in \operatorname{core}(\mathcal{C})$ is determined by $r$ binary choices; in each connected component of $G, C$ must contain exactly one of the two parts of the bipartition. This allows a more concise representation of the core. For each $C \in \operatorname{core}(\mathcal{C})$, define $p_{C} \in\{0,1\}^{r}$ such that

$$
\left(p_{C}\right)_{i}= \begin{cases}0 & \text { if } C \cap\left(U_{i} \cup V_{i}\right)=V_{i} \\ 1 & \text { if } C \cap\left(U_{i} \cup V_{i}\right)=U_{i}\end{cases}
$$

Let $S:=\left\{p_{C}: C \in \operatorname{core}(\mathcal{C})\right\} \subseteq\{0,1\}^{r}$. Then core $(\mathcal{C})$ is a duplication of cuboid $(S)$.

Claim 1 core $(\mathcal{C})$ is a tangled clutter, so (1) holds.

Proof of Claim. As a subset of $\mathcal{C}, \operatorname{core}(\mathcal{C})$ has covering number at most two, and every element of it appears in a cover of cardinality two. Thus, to prove the claim, it suffices to show that core $(\mathcal{C})$ has covering number at least two. Let $y$ be a fractional packing of $\mathcal{C}$ of value two. Then $\operatorname{support}(y) \subseteq \operatorname{core}(\mathcal{C})$ by Proposition 20 (1), so $y$ is also a fractional packing of core $(\mathcal{C})$. Subsequently, core $(\mathcal{C})$ has covering number at least two, as required.

We know that

$$
\left\{\chi_{C}: C \in \operatorname{core}(\mathcal{C})\right\}=\left\{\chi_{C}: C \in \mathcal{C}\right\} \cap\left\{x: x_{u}+x_{v}=1,\{u, v\} \in E\right\} .
$$

Claim $2 \operatorname{conv}\left\{\chi_{C}: C \in \operatorname{core}(\mathcal{C})\right\}=Q(b(\mathcal{C})) \cap\left\{x: x_{u}+x_{v}=1,\{u, v\} \in E\right\}$.

Proof of Claim. $(\subseteq)$ follows immediately from $(\star)$. () Pick a point $x^{\star}$ in the set on the right-hand side. Clearly, $x^{\star} \in Q(b(\mathcal{C}))$. Since $\mathcal{C}$ is ideal, so is $b(\mathcal{C})$, implying that for some $\lambda \in \mathbb{R}_{+}^{\mathcal{C}}$ with $\sum_{C \in \mathcal{C}} \lambda_{C}=1$, we have that

$$
x^{\star} \geq \sum_{C \in \mathcal{C}} \lambda_{C} \chi_{C} .
$$

Since for all $\{u, v\} \in E$, we have that $x_{u}^{\star}+x_{v}^{\star}=1$ and $\{u, v\} \in b(\mathcal{C})$, equality must hold above and by $(\star)$, if $\lambda_{C}>0$ then $C \in \operatorname{core}(\mathcal{C})$. Hence, $x^{\star} \in \operatorname{conv}\left\{\chi_{C}: C \in \operatorname{core}(\mathcal{C})\right\}$, as required. 
For each $i \in[r]$, pick $u_{i} \in U_{i}$ and $v_{i} \in V_{i}$, and let $\mathcal{C}^{\prime}$ be the clutter over ground set $\left\{u_{1}, v_{1}, \ldots, u_{r}, v_{r}\right\}$ obtained from core $(\mathcal{C})$ after contracting $V-\left\{u_{1}, v_{1}, \ldots, u_{r}, v_{r}\right\}$. Notice that $\left|\left\{u_{i}, v_{i}\right\} \cap C\right|=1$ for all $i \in[r]$ and $C \in \mathcal{C}^{\prime}$. Observe that $\mathcal{C}^{\prime}$ is nothing but cuboid $(S)$. $^{1}$

Claim $3 \operatorname{conv}\left(\left\{\chi_{C}: C \in \mathcal{C}^{\prime}\right\}\right)$ can be described by $z \geq \mathbf{0}, z_{u_{i}}+z_{v_{i}}=1, i \in[r]$, and

$$
\sum_{B \cap U_{i} \neq \emptyset}\left|B \cap U_{i}\right| z_{u_{i}}+\sum_{B \cap V_{j} \neq \emptyset}\left|B \cap V_{j}\right| z_{v_{j}} \geq 1
$$

for every $B \in b(\mathcal{C})$ such that for each $i \in[r], B \cap\left(U_{i} \cup V_{i}\right) \subseteq U_{i}$ or $V_{i}$. Thus, (2) holds.

Proof of Claim. Observe that $\operatorname{conv}\left\{\chi_{C}: C \in \mathcal{C}^{\prime}\right\}$ is the projection of $\operatorname{conv}\left\{\chi_{C}: C \in\right.$ core $(\mathcal{C})\}$ onto the coordinates $\left\{u_{i}, v_{i}: i \in[r]\right\}$. Thus, to give a description for $\operatorname{conv}\left\{\chi_{C}: C \in \mathcal{C}^{\prime}\right\}$, we may apply Fourier-Motzkin Elimination to the description of $\operatorname{conv}\left\{\chi_{C}: C \in \operatorname{core}(\mathcal{C})\right\}$ given by Claim 2, thereby giving us the claimed description.

Claim $4 \operatorname{conv}(S)$ is defined by $\mathbf{0} \leq x \leq \mathbf{1}$, and

$$
\sum_{B \cap U_{i} \neq \emptyset}\left|B \cap U_{i}\right| x_{i}+\sum_{B \cap V_{j} \neq \emptyset}\left|B \cap V_{j}\right|\left(1-x_{j}\right) \geq 1
$$

for every minimal cover $B$ of $\mathcal{C}$ such that for each $i \in[r], B \cap\left(U_{i} \cup V_{i}\right) \subseteq U_{i}$ or $V_{i}$. Thus, (3) holds.

Proof of Claim. This follows from Claim 3 by another application of Fourier-Motzkin Elimination.

Claim 4 finishes the proof.

Open Access This article is licensed under a Creative Commons Attribution 4.0 International License, which permits use, sharing, adaptation, distribution and reproduction in any medium or format, as long as you give appropriate credit to the original author(s) and the source, provide a link to the Creative Commons licence, and indicate if changes were made. The images or other third party material in this article are included in the article's Creative Commons licence, unless indicated otherwise in a credit line to the material. If material is not included in the article's Creative Commons licence and your intended use is not permitted by statutory regulation or exceeds the permitted use, you will need to obtain permission directly from the copyright holder. To view a copy of this licence, visit http://creativecommons.org/licenses/by/4.0/.

\section{References}

1. Abdi, A., Cornuéjols, G.: A min-max theorem for clean tangled clutters. Submitted, 2022

\footnotetext{
${ }^{1}$ Up to here, our proof is almost identical to the proof of Theorem 22, except that the statement of Claim 2 has been slightly revised to a more convenient formulation.
} 
2. Abdi, A., Cornuéjols, G., Guričanová, N., Lee, D.: Cuboids, a class of clutters. J. Comb. Theory Ser. B 142, 144-209 (2020)

3. Abdi, A., Cornuéjols, G., Huynh, T., Lee, D.: Idealness of k-wise intersecting families. Math. Program. (2020)

Publisher's Note Springer Nature remains neutral with regard to jurisdictional claims in published maps and institutional affiliations. 